ИЗВЕСТИЯ АКАДЕМИИ НАУК ЭСТОНСКОИ ССР. ТОМ Х СЕРИЯ ФИЗИКО-МАТЕМАТИЧЕСКИХ И ТЕХНИЧЕСКИХ НАУК. 1961, № 3

\title{
О ГЕОМЕТРИИ РОТОРА С ПЕЧАТНОЙ ОБМОТКОЙ В ЭЛЕКТРИЧЕСКИХ МАШИНАХ
}

\section{В. ТАЕЛ}

В последнее время в серийном производстве вращающихся электрических машин обращается все большее внимание на применение печатных схем $\left[{ }^{1-4}\right]$. Использование последних в производстве электрических машин дает возможность полностью автоматизировать производственный процесс, позволяет создать компактные конструкции, а также придать машинам ряд таких весьма ценных эксплуатационных свойств, как малая механическая постоянная времени, малая индуктивность роторной цепи и. т. п., которые делают их во многих случаях незаменимыми. Ввиду этого можно предполагать, что применение печатных схем во вращающихся электрических машинах будет все более расширяться.

В настоящее время печатные схемы используются наиболее широко в малых торцовых коллекторных машинах с постоянными магнитами. Принципиально печатные схемы могут быть применены и в более мощных машинах, особенно из-за благоприятных условий коммутации. Пример принципиальной конструкции подобной машнны приведен на фиг. 1. Магнитная цепь ее отличается от обычной, кроме относительно
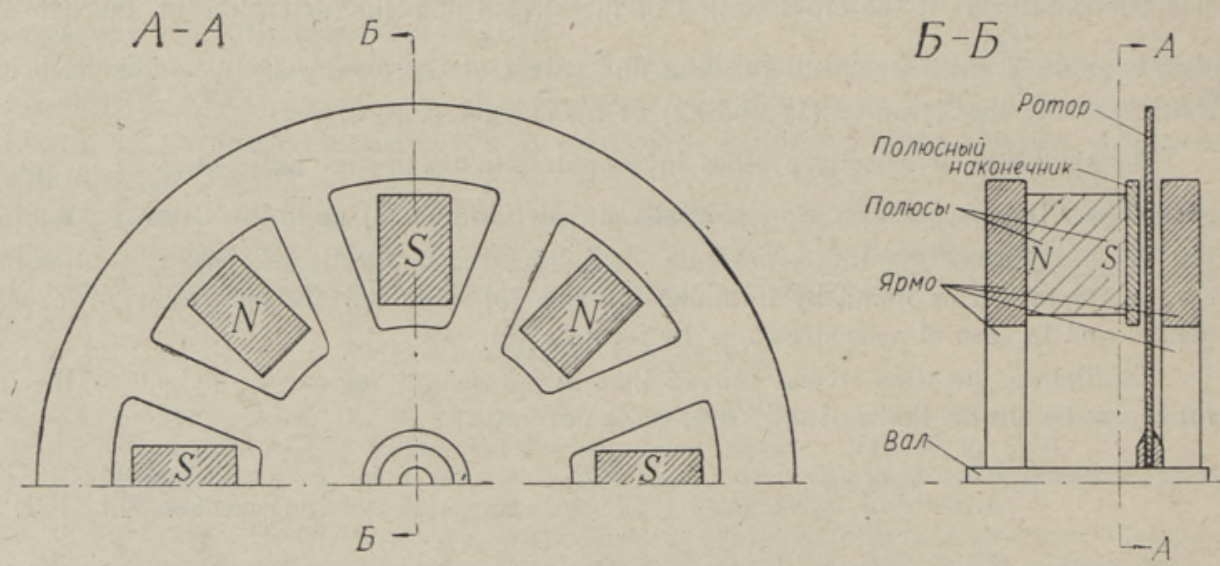

Фиг. 1.

большего воздушного зазора, еще и тем, что она свободна от вращающихся ферромагннтных частей. Якорь представляет собой насаженный на вал диск, изготовленный из фольгированной с обеих сторон медью диэлектрической пластинки, на которой фотохимическим способом образована обмотка с радиальными проводниками. Коллектором служит поверхность обмотки, по которой непосредственно скользят щетки. 
Описанные машины из-за новизны роторной конструкции обладают, по сравнению с обычными машинами, рядом особенностей, которые необходимо учитывать при проектнровании и которые до сих пор в технической литературе освещены очень слабо.

Исходя из вышеизложенного, настоящая статья ставит своей задачей дать некоторые основы для выбора части конструктивных величин ротора, имея в виду максимальное использование меди. Анализ проведен в основном для машин постоянного тока, но некоторые выводы имеют и более общий характер.

\section{Форма лобовых соединений}

Поверхность ротора, занимаемую обмоткой, можно разделить на следующие части (см. фиг. 3б):

1) внутренние лобовые соединения между радиусами $R_{1}$ и $R_{2}$;

2) активные части проводников между радиусами $R_{2}$ и $R_{3}$;

3) внешние лобовые соединения между радиусами $R_{3}$ и $R_{4}$.

Рассмотрим теперь форму лобовых соединений, учитывая равномерную толщину меди на поверхности ротора.

Равномерное использование меди, т. е. постоянная плотность тока по длине лобовых соединений, получается при постоянной ширине проводника. Так как при $N>100$ практически $\sin \frac{4 \pi}{N}=\frac{4 \pi}{N}$, то из соотношения

$$
\begin{aligned}
b_{0} & =R_{0} \frac{4 \pi}{N}= \\
& =R \frac{4 \pi}{N} \cos \alpha
\end{aligned}
$$

(где $b_{0}$ - суммарная ширина проводника и изоляционного промежутка в лобовой части; $R_{0}-$ минимальный радиус лобовых соединений; $N$ число всех проводников на диске; $R$ - произвольно выбранный радиус, при условии $\infty>R>R_{0}$; $\alpha$ - угол наклона проводника относительно радиуса-вектора на расстоя-

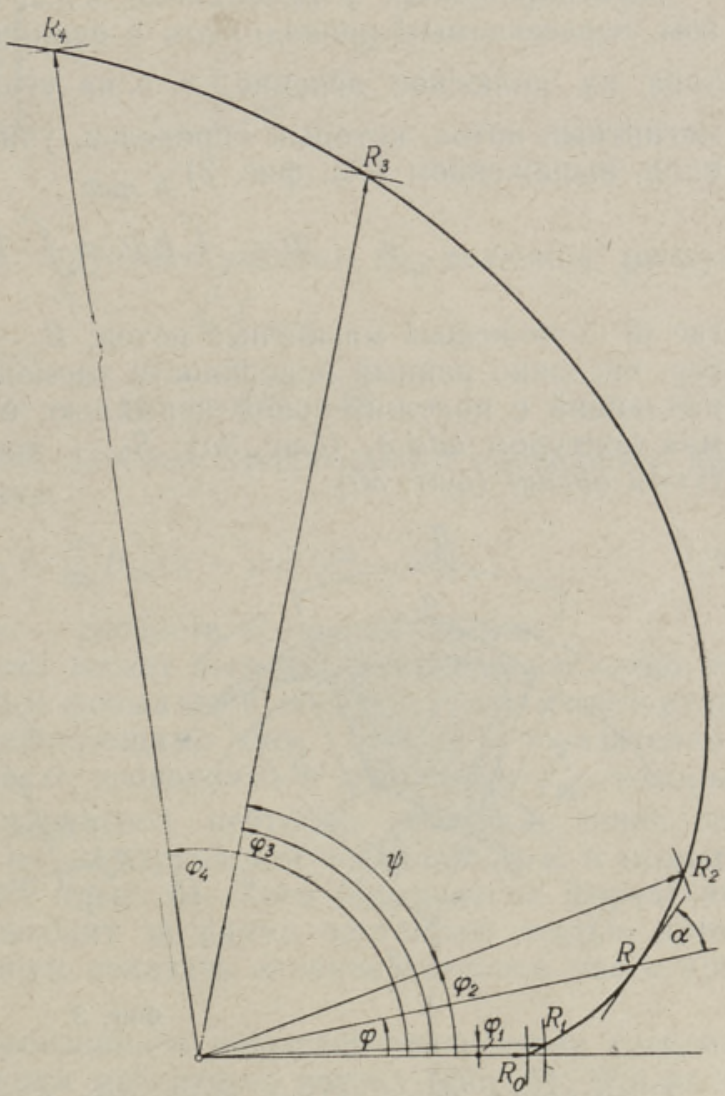

Фиг. 2. нии $R$ от центра диска) определяем закономерность

$$
\cos \alpha=\frac{R_{0}}{R} \text {. }
$$


Вышеприведенное позволяет выразить в полярных координатах общее уравнение для оси проводника лобовых соединений при условии $\infty>R>R_{0}$ :

$$
\varphi=\int_{R_{0}}^{R} \frac{\tan a}{R} d R=\tan \alpha-\alpha=\tan \arccos \frac{R_{0}}{R}-\arccos \frac{R_{0}}{R} .
$$

Кривая, полученная по уравнению (3), показана на фиг. 2. Таким образом, осевая линия проводника лобовых соединений машины определяется упомянутой кривой:

1) для внутренних частей от $R_{1}$ до $R_{2}$ и

2) для внешних частей от $R_{3}$ до $R_{4}$.

Забегая вперед, отметим, что на фиг. 2 показаны величины радиусов; соответствующие обмотке, виток которой приведен далее на фиг. 76.

\section{Электродвижущая сила}

Индуцированная в проводнике э.д.с. определена магнитным потоком, пересекаемым проводником в единицу времени. При повороте ротора на полюсное деление, т. е. на угол $\frac{\pi}{p}$, проводник пересекает магнитный поток, который определен, учитывая симметрию магнитного поля, выражением (см. фиг. 3)

$$
\Phi=\int_{S_{1}} B d s=\int_{S_{2}} B d s
$$

где Ф - полезный магнитный поток; $B$ - вектор индукции; $d s-$ вектор, численно равный поверхности элемента $d s$ и по направлению совпадающий с положительной нормалью; $S_{1}$ - поверхность, охватываемая контуром $a d d_{1} a_{1}$ (фиг. $\left.3 a\right) ; S_{2}$ - поверхность, охватываемая контуром abcdef (фиг. 3б).
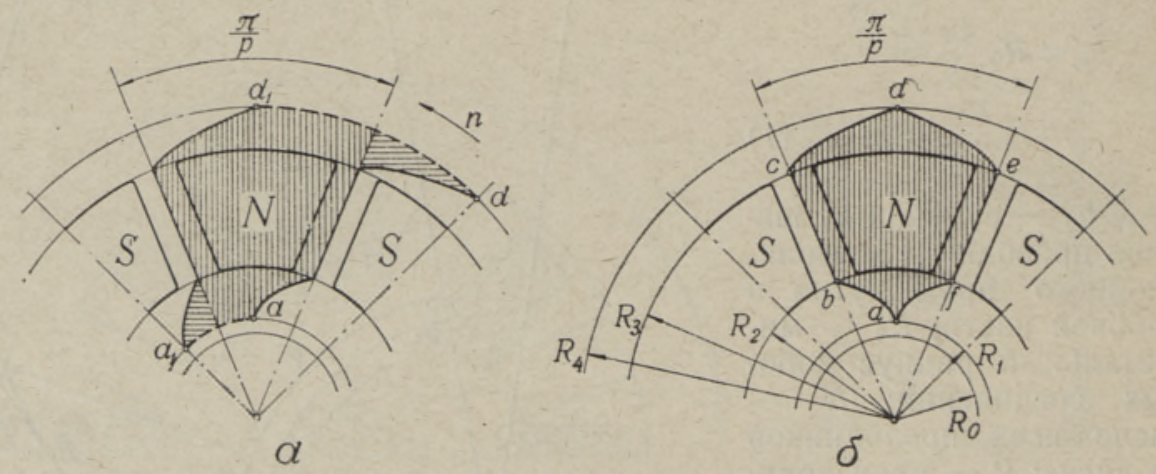

Фиг. 3.

Суммарная поверхность полюсных наконечников машины, которую мы условно назовем активной поверхостью машины $S_{\text {акт }}$, определена выражением

$$
S_{\mathrm{akr}}=(\pi-p x)\left(F_{3}^{2}-R_{2}^{2}\right),
$$

где $x$ - угол, который возник бы между боковыми образующими со- 
седних полюсных наконечников, если бы эти образующие были радиальными, при неизменной активной поверхности (фиг. 4).

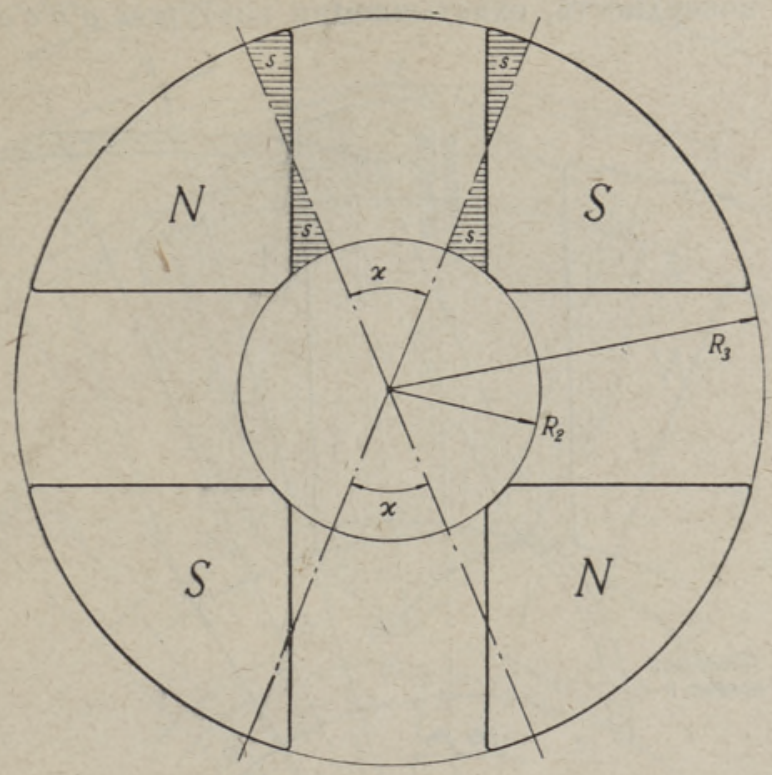

Фиг. 4.

Назовем условно средней индукцией машины $B_{\text {ср }}$ величину, равную

$$
B_{\mathrm{cp}}=\frac{2 p \Phi}{S_{\text {aкт }}}=\frac{2 p \int_{S_{2}} B d s}{(\pi-p \%)\left(R_{3}^{2}-R_{2}^{2}\right)} .
$$

Итак, получаем выражение для э.д.с. радиального проводника при постоянной скорости вращения

$$
e=\frac{n}{60} B_{\mathrm{cp}} S_{\text {акт }}=\frac{n}{60} B_{\mathrm{cp}}(\pi-p \varkappa)\left(R_{3}^{2}-R_{2}^{2}\right),
$$

где $e-$ э.д.с. проводника; $n-$ скорость вращения, об/мин.

Активная часть проводника может быть расположена наклонно относительно радиуса-вектора, и форма ее в общем случае может отличаться от прямой. Характеризующими скос (наклон) показателями являются форма активной части проводника и углы $\gamma_{R_{2}}$ и $\gamma_{R_{3}}$, которые определяют относительно магнитной нейтрали конечные точки оси активной части проводника на электрической нейтрали, т. е. в момент, когда э.д.с. проводника $e=0$ (фиг. 5). Хотя определение формы активной части проводника выходит за рамки настоящей статьи, автор все же сцитает целесообразным показать влияние активной части проводника на э.д.с.

Скос активной части проводников вызывает уменьшение сцепленного с проводниковым контуром магнитного потока (фиг. 6). Это явление характеризуется коэффициентом скоса

$$
\xi=\frac{\int_{s_{3}} B d s}{\int_{S_{2}} B d s},
$$


где $\xi$ - коэффициент, учитываюший уменьшение магнитного потока в результате наклона проводников (в случае радиальных проводников $\xi=1) ; S_{3}$ - поверхность, охватываемая контуром $a^{\prime} b^{\prime} c^{\prime} d^{\prime} e^{\prime} f^{\prime}$ (фиг. 6).

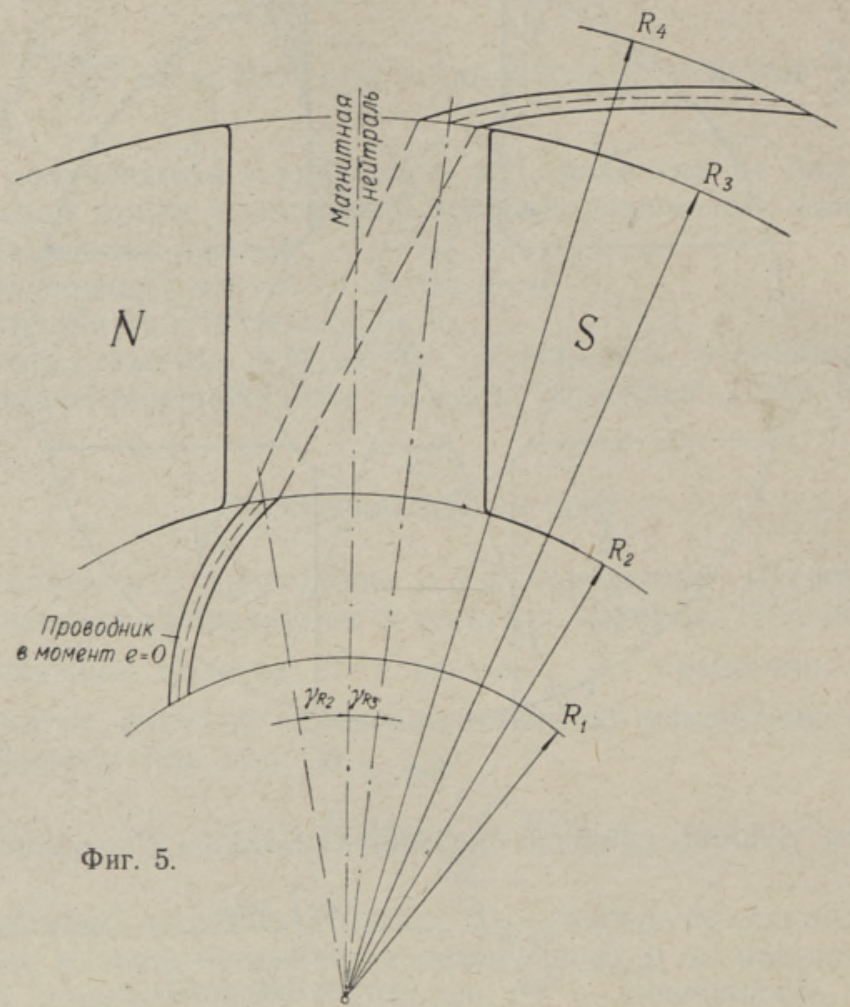

Коэффициент $\xi$ зависит в основном от распределения магнитного поля в воздушном зазоре и от наклона активных частей проводников.

Итак, можем написать выражение для суммарной э.д.с. машины

$$
E=\frac{N}{2 a} \frac{n}{60} B_{\mathrm{cp}} \xi(\pi-p \varkappa)\left(R_{3}^{2}-R_{2}^{2}\right) .
$$

Здесь $E-$ э.д.с.' машины; $\frac{N}{2 a}$ - число проводников в параллельной ветви.

В выражение э.д.с. машины вошла возможная ошибка, связанная с разностью магнитных потоков, сцепленных проводниковыми контурами по обе стороны диска, зависящая от толщины диэлектрической основы диска и от конфигурации магнитной системы.

\section{Мощность}

Внутренняя электромагнитная мощность машины

$$
P^{\prime}=E I \text {. }
$$

При условии, что ширина активной части проводника $b_{\text {акт }} \geqslant b$ ток проводника определяется формулой

$$
I_{\text {пр }}=\delta h b=\delta h R_{0} \frac{4 \pi}{N} \lambda_{1}=\delta h R_{2} \frac{4 \pi}{N} \lambda_{1} \cos \alpha_{2},
$$




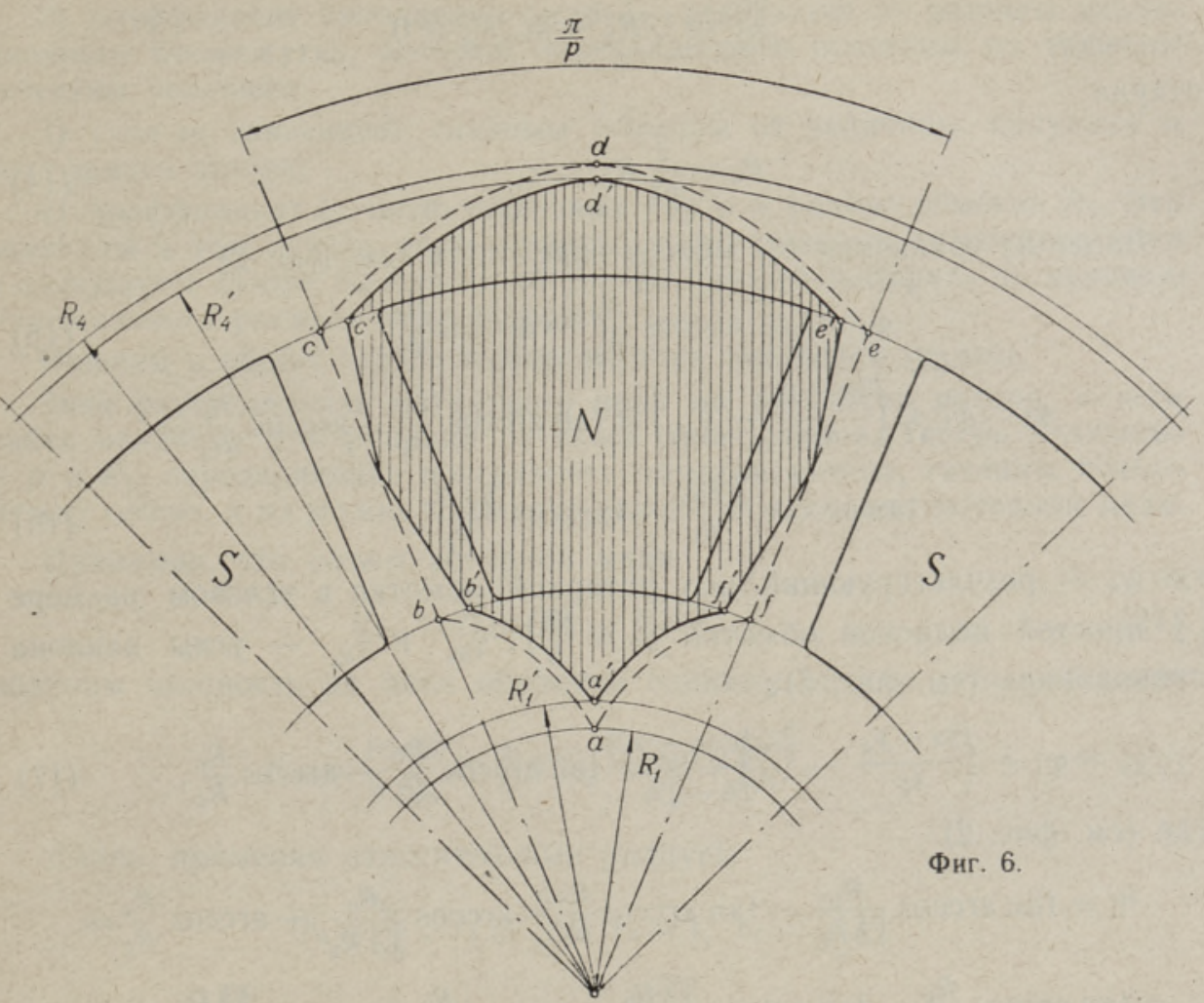

где $I_{\text {пр }}$ - ток проводника; $\delta$ - допустимая плотность тока; $h-$ толщина меди; $b$ - ширина проводника в лобовых соединениях; $\lambda_{1}=1-\frac{b_{\text {из }} N}{4 \pi R_{0}} \quad-$ коэффициент заполнения медью проводника лобо-

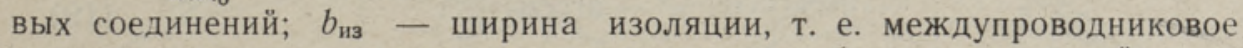
расстояние; $\alpha_{2}$ - угол наклона проводника лобовых соединений относительно радиуса-вектора на расстоянии $R_{2}$ от центра диска.

Так как суммарный ток машины

$$
I=2 a I_{\text {пр }},
$$

то ее внутренняя электромагнитная мощность (не учитывая возможное влияние реакции якоря) равна

$$
P^{\prime}=\frac{\pi}{15} n \delta B_{\mathrm{cp}} h \lambda_{1} \cos \alpha_{2} \xi(\pi-p \varkappa)\left(R_{2} R_{3}^{2}-R_{2}^{3}\right) .
$$

\section{Соотношения между радиусами обмотки}

Из выражения (13) можем при условии максимума определить соотношение между $R_{2}$ и $R_{3}$, допуская, что величина

$$
C^{\prime}=\frac{\pi}{15} n \delta B_{\mathrm{cp}} h \lambda_{1} \cos \alpha_{2} \xi(\pi-p \varkappa)
$$

не зависит от $R_{2}$. 


$$
\frac{\partial P^{\prime}}{\partial R_{2}}=C^{\prime} R_{3}^{2}-3 C^{\prime} R_{2}^{2}=0
$$

откуда

$$
R_{2}=\frac{1}{\sqrt{3}} R_{3}
$$

Далее из уравнения (3) можем определить (см. фиг. 2)

$$
\begin{gathered}
\varphi_{1}=\tan \arccos \frac{R_{0}}{R_{1}}-\arccos \frac{R_{0}}{R_{1}} \\
\varphi_{2}=\varphi_{1}+\left(\frac{2 \pi-y_{k}}{4 p}-\gamma_{R_{2}}\right)=\tan \arccos \frac{R_{0}}{R_{2}}-\arccos \frac{R_{0}}{R_{2}}= \\
=\tan \arccos \frac{\sqrt{3} R_{0}}{R_{3}}-\arccos \frac{\sqrt{3} R_{0}}{R_{3}},
\end{gathered}
$$

где $y_{k}$ - результирующий шаг роторной обмотки в угловом размере (у простой волновой обмотки $\left.y_{k}=\frac{4 \pi}{N}\right) ; v_{R_{2}}$ и $\gamma_{R_{2}}-$ углы наклона проводников (см. фиг. 5).

$$
\varphi_{3}=\varphi_{1}+\left(\frac{2 \pi-y_{k}}{4 p}-v_{R_{2}}\right)+\Psi=\tan \arccos \frac{R_{0}}{R_{3}}-\arccos \frac{R_{0}}{R_{3}},
$$

где (см. фиг. 2)

$\Psi=\tan \arccos \frac{R_{0}}{\sqrt{3} R_{2}}-\tan \arccos \frac{R_{0}}{R_{2}}-\arccos \frac{R_{0}}{\sqrt{3} R_{2}}+\arccos \frac{R_{0}}{R_{2}}=$

$=\tan \arccos \frac{R_{0}}{R_{3}}-\tan \arccos \frac{\sqrt{3} R_{0}}{R_{3}}-\arccos \frac{R_{0}}{R_{3}}+\arccos \frac{\sqrt{3} R_{0}}{R_{3}} ;$

$$
\begin{gathered}
\varphi_{4}=\varphi_{1}+\left(\frac{2 \pi-y_{k}}{4 p}-\gamma_{R_{2}}\right)+\Psi+\left(\frac{2 \pi-y_{k}}{4 p}-\gamma_{R_{3}}\right)= \\
=\tan \arccos \frac{R_{0}}{R_{4}}-\arccos \frac{R_{0}}{R_{4}} .
\end{gathered}
$$

Среди представленных соотношений имеем три уравнения (15), (17) и (19), содержащие пять неизвестных $\varphi_{1}, R_{0}, R_{1}, R_{3}$ и $R_{4}$. Так как $R_{3}$ определяется мощностью машины, то для решения этой системы необходимо найти одно дополнительное условие. Таковым должен служить экономический критерий $K_{\text {эк }}=$ макс, который выведен в следующем подразделе.

\section{Анализ выражения мощности}

Учитывая соотношения (2) и (14), получим выражение для мощности

$$
P^{\prime}=\frac{2 \pi}{45} n \delta B_{\mathrm{cp}} h \lambda_{1} \xi(\pi-p \varkappa) R_{0} R_{3}^{2} .
$$

Анализируя эту формулу, видим, что при данном радиусе $R_{3}$ величины, определяющие мощность машины на один оборот, можно подразделить следующим образом:

1) удельные нагрузки $\delta B_{\text {ср }}$ и толщина меди $h$. Выбираются по тепловому расчету и распределению потерь всей машины; 
2) коэффициент заполнения меди $\lambda_{1}$, зависящий от ширины изоляционного промежутка, который определяется в основном по технологическим условиям;

3) угол $x$, зависящий главным образом от величины индукции и воздушного зазора;

4) коэффициент $\xi$, число полюсных пар $p$ и радиус обмотки $R_{0}$. Эти величины в том случае, если известно распределение магнитного поля в воздушном зазоре на плоскости ротора, можно выбирать по условию минимального расхода меди ротора.

Опишем в общих чертах выбор величин последней группы.

Зная распределение магнитного поля на плоскости ротора, можем найти величину $\xi$ в функции от скоса проводников. Теперь величины $\xi, p$ и $R_{0}$, определяющие поверхность обмотки ротора, связаны только между собою, и их можно выбирать исходя из экономии роторной меди.

Принимая, что объем роторной меди

$$
V_{\mathrm{pм}} \approx 2 \lambda_{1} \pi\left(R_{4}^{2}-R_{1}^{2}\right) h,
$$

получим мощность на один оборот и единицу объема роторной меди

$$
K_{\text {эк }}=\frac{P^{\prime} \cdot 60}{n V_{\mathrm{pM}}} \approx \frac{4}{3} \delta B_{\mathrm{cp}} \frac{\xi(\pi-p \varkappa) R_{0} R_{3}^{2}}{R_{4}^{2}-R_{1}^{2}} .
$$

Далее, применяя относительные радиусы

$$
r_{0}=\frac{R_{0}}{R_{3}}, r_{1}=\frac{R_{1}}{R_{3}}, r_{3}=1, r_{4}=\frac{R_{4}}{R_{3}},
$$
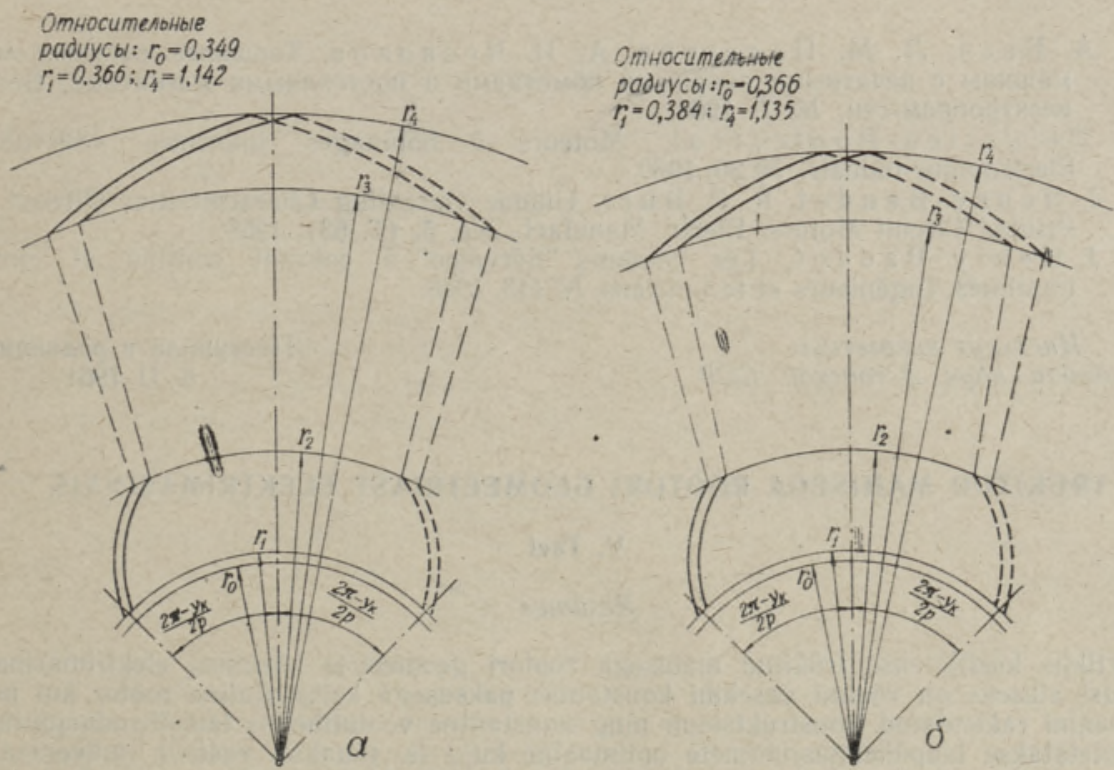

Фиг. 7.

мы можем при выборе скоса проводников (коэффициент छ), числа полюсных пар $p$ и относительного радиуса $r_{0}$ пользоваться переменной частью выражения (22) как экономическим критерием 


$$
K_{\text {эк }} \equiv \frac{\xi(\pi-p \varkappa) r_{0}}{r_{4}^{2}-r_{1}^{2}}=\text { макс, }
$$

если это не противоречит особым техническим требованиям.

В качестве примера на фиг. 7 приведены формы витка роторной обмотки, соответствующие выбранным по критерию (23) относительным радиусам и параметрам $N=194, p=4$, при а) радиальных проводниках и б) скосе $\gamma_{R_{2}}+\gamma_{R_{3}}=\frac{6 \pi}{N}$, причем $\gamma_{R_{2}}=\frac{R_{3}}{R_{2}} \gamma_{R_{3}}$.

Из приведенных на фиг. 6 и 7 построений вытекает, что небольшой скос проводников (коэффициент $\xi \approx 1$ ), влияя на $r_{1}$ и $r_{4}$, дает экономию меди.

\section{Выводы}

1. Форма лобовых соединений в дискообразном роторе определяется универсальной кривой (3).

2. Мощность машины зависит от отношения радиусов $\frac{R_{3}}{R_{9}}$ и при заданном радиусе $R_{3}$ и угле $x$ достигает максимума при $R_{2}=\frac{R_{3}}{\sqrt{3}}$.

3. Численные величины $\xi, p$ и $r_{0}$, влияющие на мощность машины, могут быть выбраны исходя из экономии роторной меди, т. е. по максимальной мощности на один оборот и единицу объема роторной меди.

\section{ЛИТЕ РА ТУРА}

1. В. А. Баев, Л. М. П ал астин, А. И. Чесноков, Торцовые регулируемые машины с печатными якорными обмотками и постоянными магнитами, Вестн. электропром-сти, № 7, 1960.

2. P. Dejussieu-Pontcarral, Moteurs à bobinages imprimés «Sérvalco», Ëlectronique industr., № 30, 1960.

3. M. J. Henry-B a ud ot, R. P. B urr, Unique Operating Characteristics Offered by Printed Circuit Motors, Electr. Manufact., No. 5, (T. 63), 1959.

4. M. J. Hen ry-B a u dot, Les moteurs "Sérvalco" à courant continu et circuit imprimés, Ingénieurs et techniciens, № 113, 1958.

Ннститут энергетики

Академии наук Эстонской ССР
Поступила в редакцию

8. II 1961

\section{TRUKITUD MÄHISEGA ROOTORI GEOMEETRIAST ELEKTRIMASINAIS}

\section{Tael \\ Resümee}

Artiklis käsitletakse trükitud mähisega rootori geomeetria küsimusi elektrimasinais. Analüüsi aluseks on võetud vasekihi konstantse paksusega kettakujuline rootor kui peamine senini rakendatud konstruktsioon ning konstantne voolutihedus laupühendusjuhtmetes. Tuletatakse laupühendusjuhtmete optimaalne kuju ja tuuakse vastava universaalse kôvera avaldis polaarkoordinaatides. Tuletatakse avaldised alalisvoolumasina elektromotoorsele jōule ja võimsusele. Lähtudes vōimsuse maksimumist, leitakse mähise aktiivosa raadiuste optimaalne suhe. Näidatakse, et osa alalisvoolumasina vōimsust mōjutavaid suurusi, nagu juhtmete kallutuse, pooluspaaride arvu ja rootorimähise minimaalse raadiuse võib valida rootori vase ökonoomsusest lähtudes. 


\section{ON THE GEOMETRY OF ROTORS WITH PRINTED COILS IN ELECTRIC MACHINES}

\section{Tael}

\section{Summary}

The author deals with the problems of rotors with printed coils in electric machines. For the basis of analysis a disc-shaped rotor with a constant thickness of copper layer has been taken, as well as a condenser of current in frontal connections. The optimal shape of frontal connections is computed, and an equation of the corresponding universal curve in polar coordinates is given. Equations of the electromotive power and capacity of a direct current machine are derived. Proceeding from the maximum capacity, the optimal relation of the radii of the active part of the coils is found. It is shown that a part of the factors influencing the direct current machines, as the incline of wires, number of polar pairs and the minimal radius of the rotor coil can be chosen with the consideration of the economy of rotor copper.

\author{
Academy of Sciences of the Estonian S.S.R. \\ Institute of Energetics
}

Received

February 8th, 1961 